\title{
OPEN Entropy optimization and heat transfer analysis in MHD Williamson nanofluid flow over a vertical Riga plate with nonlinear thermal radiation
}

\author{
Muhammad Rooman ${ }^{1,2}$, Muhammad Asif Jan ${ }^{1}$, Zahir Shah ${ }^{2 \bowtie}$, Poom Kumam ${ }^{3,4 \bowtie ~ \& ~}$ \\ Ahmed Alshehri ${ }^{5}$
}

The entropy generation for a reactive Williamson nanofluid flow past a vertical Riga system is the subject of this article. The effects of MHD, thermophoresis, nonlinear heat radiation and varying heat conductivity are modeled into the heat equation in the established model. Suitable similarity transformations are examined to bring down the partial differential equations into ordinary differential equations. The Homotopy analysis approach is used to solve the dimensionless transport equations analytically. The graphic information of the various parameters that emerged from the model is effectively collected and deliberated. The temperature field expands with thermophoresis, Brownian motion and temperature ratio parameters as the modified Hartmann number forces an increase in velocity, according to the findings of this analysis. With the increase in the fluid material terms, the entropy generation and Bejan number increase. Riga plate has numerous applications in improving the thermo-physics features of a fluid, the value of magnetic field embraces an important role in fluid mechanics. An external electric field can be used to control flow in weak electrically conductive fluids. The Riga plate is one of the devices used in this regard. It's a device that creates electromagnetic fields. They produce the Lorentz force which is a force that directs fluid flow. The authors have discussed the entropy optimization for a reactive Williamson nanofluid flow past a vertical Riga plate is addressed. This is the first investigation on mass and heat transfer flow that the authors are aware of, and no similar work has yet been published in the literature. A thorough mathematical examination is also required to demonstrate the model's regularity. The authors believe that the results acquired are novel and have not been plagiarized from any other sources.

Modern research has discovered that high-profile freezing is compulsory for the majority of industrial and technological operations. By deprived thermo-physical properties of the typical fluid, the extraordinary heat mass flux efficiency is difficult to achieve. The issue was talked to a certain amount by the overview of the notion of nanoliquids. A nanoliquid is a liquid drenched by extremely conductive metallic particles of Nano-size. As compared to larger particles, nanoparticles will easily remain suspended in the base liquid for an extended period of time. Following Choi ${ }^{1}$ research and corresponding reviews, nanofluids appear to be organizations encouraged in mass and heat transfer applications, specifically in heat exchange, aerospace technology, micro processing, refrigeration and automotive, etc. in which extremely energetic products are elaborated with both a slight size and compressed form. Nanoliquids are also significant from a medical standpoint. The applications of nanofluids

\footnotetext{
${ }^{1}$ Institute of Numerical Sciences, Kohat University of Science and Technology KUST, Kohat 26000, Khyber Pakhtoonkhwa, Pakistan. ${ }^{2}$ Department of Mathematical Sciences, University of Lakki Marwat, Lakki Marwat 28420, Khyber Pakhtunkhwa, Pakistan. ${ }^{3}$ Fixed Point Research Laboratory, Fixed Point Theory and Applications Research Group, Center of Excellence in Theoretical and Computational Science (TaCS-CoE), Faculty of Science, King Mongkut's University of Technology Thonburi (KMUTT), 126 Pracha Uthit Rd., Bang Mod, Thung Khru, Bangkok 10140, Thailand. ${ }^{4}$ Department of Medical Research, China Medical University Hospital, China Medical University, Taichung 40402, Taiwan. ${ }^{5}$ Department of Mathematics, Faculty of Sciences, King Abdulaziz University, Jeddah 21589, Saudi Arabia. ${ }^{\circledR}$ email: zahir@ulm.edu.pk; poom.kum@kmutt.ac.th
} 
in medical field are the treatment of hyperthermia, therapy for cancer care, surgeries, openings and wounds/ blocking the veins etc. Buongiorno ${ }^{2}$ presented the knowledge of nanoliquid and established a mathematical model to analyze and explore the thermal features of base fluids. Later on, various expansions have been made in the nanofluids field. Mustafa et al. ${ }^{3}$ founded an analytical solution of nanofluid flow adjacent to a stagnation point near a stretching shallow by using HAM. Turkyilmazoglu ${ }^{4}$ founded exact analytical solution for MHD nanofluid flow for transfer of mass and heat across a porous shrinking surface. Nield and Kuznetsov ${ }^{5}$ presented an analytical solution for the problem of Cheng-Minkowycz in a medium with pores drenched by nanoliquid. The energy conservation problems of convection conjugate conduction and heat transfer radiation with magnetic effects and viscous dissipation were investigated by $\mathrm{Hsiao}^{6}$. Theoretically laminar flow and rate of hear transfer of water/alumina nanoparticles favored a rounded microchannel in the incidence of the uniform magnetic field were investigated by Malvandi and Ganji ${ }^{7}$.

Because of its numerous applications in improving the thermo-physics features of a fluid, the value of the magnetic field embraces an important role in fluid mechanics. Various liquids that are weak conductors of electricity are encountered in fields for instance earth sciences and astrology. As a result, an external agent is often required to improve phenomena of heat flow via improved conductivity and other thermo-physics properties. A magnetic piece or a forever fixed series of these magnets with discontinuous electrodes could be used as this external agent. Gailitis and Lielausis ${ }^{8}$ was the first to use this type of formulation in Riga and it was formally introduced Riga Plate. The Riga plate as constructed is much useful because it has rapidly become well-known in industrial procedures causing fluid flow behavior. Ahmed et al. ${ }^{9}$ investigated the effects of surface heating through no mass flux. The effect of the Lorentz force, which decays significantly with growing movement from the plate's surface, was used in the model by using the Riga plate. Sheikholeslami et al. ${ }^{10}$ investigated the effect of Lorentz forces on nanofluid Marangoni convection. Shafiq et al. ${ }^{11}$ investigated the flow of fluid points across a Riga plate using the Walters-B model. Adeel et al. ${ }^{12}$ has examined the mixed convection of nanoparticles in a fluid flow with a vertically mounted Riga plate. Thermal radiation's effect on Marangoni convective nanoliquid flow against a Riga plate was studied by Rasool et al. ${ }^{13}$.

The most prevalent non-Newtonian fluids observed are pseudoplastic fluids. Because of its broad collection of applications in industry, polymers with a high molecular weight melt, emulsion of coated sheets such as photographic films, polymer layer extrusion, and so on are examples; the analysis of the pseudoplastic fluid flow at the boundary layer is of keen importance. The Navier Stokes equations by themselves are insufficient to describe fluid rheological characteristics. As a consequence, rheological models have been suggested to resolve this shortcoming. Many models are being suggested to describe the Structure of pseudoplastic fluids, including the Carreau model, power-law model, Ellis fluid model, and Cross model, but Williamson fluid model has received slight attention.

The Williamson fluid is one the most essential non-Newtonian fluids having reduced viscosity as shear stress rises and features that are quite comparable to polymeric solutions. In other words, in the Williamson fluid, the functional viscosity should decrease forever as the shear rate rises with infinite viscosity at rest and nil viscosity as the shear rate approaches infinity. Williamson ${ }^{14}$ studied the pseudoplastic flow substances and formulated a model equation to explain the pseudoplastic fluids flow, which he then tested experimentally. The movement of a tinny film of a Williamson fluid by an inclined surface by means of a gravitational field was investigated by Lyubimov and Perminov ${ }^{15}$. Williamson fluid-applied into a rock fragment perturbation solution was investigated by Dapra and Scarpi ${ }^{16}$. Nadeem et al. ${ }^{17}$ addressed the Williamson fluid of peristaltic flow. Vasudev et al. ${ }^{18}$ investigated the heat transfer effects of peristaltic thrusting of a Williamson fluid over a medium with pores. Nadeem et al. ${ }^{19}$ examined the Williamson fluid model's two-dimensional flow through a stretching sheet. Compared to other versions, this one best suits the investigational statistics polymer solutions and particle interruptions, according to Cramer et al. ${ }^{20}$. The power law theory shows that when the shear rate reaches infinity, the apparent/effective viscosity should decrease forever, resulting in at rest, the viscosity is infinite and as the shear rate increases to infinity, the viscosity is zero. A natural fluid has both higher and lower active viscosity based on the molecular nature of the fluid. Both the maximum and minimum are put into consideration in the Williamson fluid model. As a result, it will produce better results for pseudoplastic fluids. Ahmad et al. ${ }^{21}$ explored the Maxwell nanofluid transfer between two coaxially simultaneous stretchy rotating disks in the context of an axial magnetic field and varying thermal conductivity. They applied the Buongiorno nanofluid model to demonstrate upper and lower disks behavior in both the same and opposing orientations. Turkyilmazoglu ${ }^{22}$ investigated the influence of magnetic fields and slip on the flow and heat transfer of a stagnation point Jeffrey fluid on deformed objects. Abo-Elkhair et al. ${ }^{23}$ established the modest Reynolds number and imposed magnetic field impacts on the hybrid Bio-nanofluid via a peristaltic channel in the dispersion of nanoparticles such as gold and copper. Bhatti et al. ${ }^{24}$ examined the applicability of the slip occurrence in bioconvection characteristics in a non-Newtonian Eyring-Powell nanofluid model constrained by a stretching sheet. References list a number of useful and fruitful papers on nanofluid research and various approaches ${ }^{25-31}$.

Motivated from the above stated facts is to examine entropy optimization and heat transfer analysis in MHD Williamson nanofluid over a vertical Riga plate with nonlinear thermal radiation. The novel aspect of the current study is to determine irreversibility for reactive Williamson nanofluid transport over a Riga vertical porous system with nonlinear convection. The study was prompted by a number of presentations on Williamson nanoliquid flow and its relevant characteristics in engineering and manufacturing. The thermodynamic second law considers complete entropy optimization for irreversibility. The problem is explained analytically using the HAM. Different flow characteristics are investigated, and the results are graphed. 

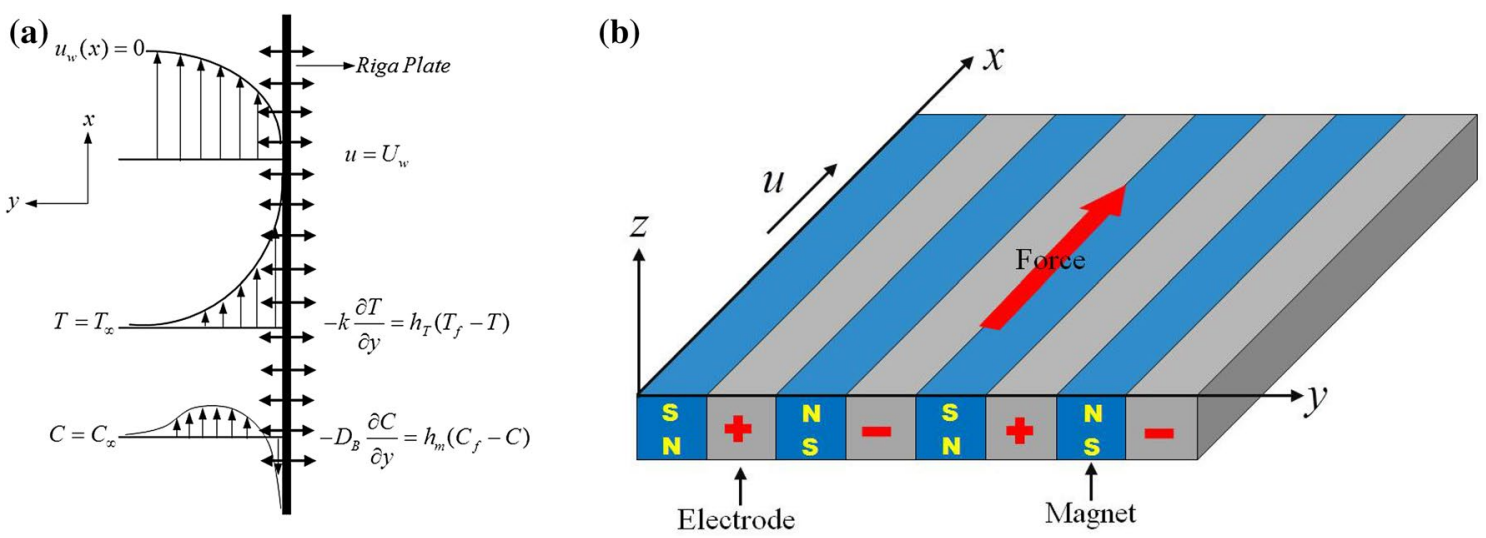

Figure 1. (a) The fluid flow geometry. (b) Lorentz force induced Riga plate.

\section{Mathematical formulation}

Consider a vertical Riga plate with a two-dimensional, steady, incompressible and reactive Williamson nanofluid flow. The plate's coordinates $(x, y)$ corresponds to the velocity components $(u, v)$ see (Fig. 1a). As shown in Fig. 1a,b the $x$-axis is aligned with the flow direction, whereas the $y$-axis is parallel to it. The Riga plate is built up of different arrays that are split into electrodes and magnets which are mounted on the board, the width of electrodes and magnets is denoted by $s$. The surface stretches non-linearly velocity $u=U_{w}=b x^{n}$ where $b$ represents the stretching rate, $U_{w}$ represents the surface velocity and $n$ is a power index. The Lorentz force is generated when the Riga plate's electromagnetic field interacts with the nanoparticles contained in the base fluid.

Magnetic hydrodynamics (MHD) is used in this issue (Fig. 1b), and the effect of a higher-order chemical reaction is factored into the nanomaterial concentration equation. Meanwhile, the energy equation investigates the occurrence of nonlinear thermal radiation, thermophoresis, and Brownian diffusion. Surface mass flux, convective thermal and mass boundary conditions also have an effect on the model.

The Cauchy stress tensor $\boldsymbol{S}$ for Wiliamson fluid model is ${ }^{17}$

$$
S=-p I+\tau
$$

The concept of extra Stress $\boldsymbol{\tau}$ for Wiliamson fluid is

$$
\boldsymbol{\tau}=\left[\mu_{\infty}+\frac{\left(\mu_{0}-\mu_{\infty}\right)}{1-\Gamma \dot{\gamma}}\right] \boldsymbol{A}_{1}
$$

where $\mu_{0}$ represents limiting viscosity at zero shear rate, $\mu_{\infty}$ represents limiting viscosity at an infinite shear rate, $\boldsymbol{A}_{1}$ represents the first Rivlin-Erickson tensor, $\boldsymbol{\Gamma}>0$ is time constant and $\dot{\gamma}$ is defined as

$$
\dot{\gamma}=\sqrt{\frac{1}{2} \operatorname{trace}\left(A_{1}^{2}\right)}
$$

Here we assumed the case for which $\mu_{\infty}=0$ and $\Gamma \dot{\gamma}<1$, so by using binomial expansion Eq. (3) can be written as:

$$
\boldsymbol{\tau}=\mu_{0}[1+\Gamma \dot{\gamma}] A_{1}
$$

Modeling temperature-dependent thermal conductivity is expressed as

$$
k(T)=k_{\infty}\left(1+\delta \frac{T-T_{\infty}}{T_{f}-T_{\infty}}\right)
$$

where $k_{\infty}$ stands for thermal conductivity at room temperature and $\delta$ stands for its parameter. The derived transport equations are expressed as with the highlighted assumptions and Oberbeck-Boussinesq associated with the well-known boundary layer approximation.

$$
\begin{gathered}
\frac{\partial u}{\partial x}+\frac{\partial v}{\partial y}=0 \\
u \frac{\partial u}{\partial x}+v \frac{\partial u}{\partial y}=v \frac{\partial^{2} u}{\partial y^{2}}+\sqrt{2} v \boldsymbol{\Gamma} \frac{\partial u}{\partial y} \frac{\partial^{2} u}{\partial y^{2}}+\frac{\pi j_{0} M^{*} \exp \left(-\frac{\pi y}{s}\right)}{8 \rho_{f}}-\frac{\sigma B_{0}^{2} u}{\rho} \\
+\frac{1}{\rho_{f}}\left[\left(1-C_{\infty}\right) \rho_{f}\left(T-T_{\infty}\right) \beta_{T}-\left(\rho_{p}-\rho_{f}\right)\left(C-C_{\infty}\right)\right] g
\end{gathered}
$$




\begin{tabular}{|l|l|l|l|}
\hline Symbols & Description & Symbols & Description \\
\hline$u, v$ & Velocity in $x, y$ direction & $k$ & Thermal conductivity \\
\hline$v_{f}$ & Base fluid kinematic viscosity & $C$ & Nanoparticle concentration \\
\hline$\rho_{f}$ & Base fluid density & $F_{1}$ & Nan-uniform inertia coefficient \\
\hline$\mu$ & Fluid viscosity & $U_{w}$ & Velocity at the sheet \\
\hline$W e$ & Fluid material constant & $g$ & Acceleration due to gravity \\
\hline$T$ & Temperature & $M^{*}$ & magnetic effect \\
\hline$T_{\infty}$ & Temperature at free stream & $\beta_{T}$ & Coefficient of thermal diffusion \\
\hline$c_{p}$ & Specific heat & $C_{\infty}$ & Free stream nanoparticles concentration \\
\hline$n$ & Power index & $k_{1}$ & Chemical reaction rate \\
\hline$D_{B}$ & Brownian diffusion coefficient & $D_{T}$ & Thermophoretic diffusion coefficient \\
\hline$\rho_{p}$ & Nanoparticles density & $\left(\rho c_{p}\right)_{f}$ & Base fluid heat capacity \\
\hline$\left(\rho c_{p}\right)_{p}$ & Nanoparticles heat capacity & $j_{0}$ & Current density \\
\hline$\rho$ & Fluid density & $\sigma$ & Electrical conductivity \\
\hline$B_{0}$ & Uniform magnetic field intensity & $s$ & Breadth of magnets and electrodes \\
\hline
\end{tabular}

Table 1. Various symbols appear in the governing equations.

$$
\begin{aligned}
& \left(\rho c_{p}\right)_{f}\left(u \frac{\partial T}{\partial x}+v \frac{\partial T}{\partial y}\right)=\frac{\partial}{\partial y}\left(k(T) \frac{\partial T}{\partial y}\right)+\left(\rho c_{p}\right)_{p}\left[\frac{D_{T}}{T_{\infty}}\left(\frac{\partial T}{\partial y}\right)^{2}+D_{B}\left(\frac{\partial T}{\partial y} \frac{\partial C}{\partial y}\right)\right]+\frac{16 a^{*}}{3 b^{*}} \frac{\partial}{\partial y}\left(T^{3} \frac{\partial T}{\partial y}\right) \\
& u \frac{\partial \phi}{\partial x}+v \frac{\partial \phi}{\partial y}=D_{B} \frac{\partial^{2} C}{\partial y^{2}}+\frac{D_{T}}{T_{\infty}}\left(\frac{\partial^{2} T}{\partial y^{2}}\right)-k_{1}\left(C-C_{\infty}\right)
\end{aligned}
$$

The Boundary Conditions are listed as:

$$
\begin{gathered}
u=U_{w}=b x^{n}, v=V_{w},-k \frac{\partial T}{\partial y}=h_{1}\left(T_{f}-T\right),-D_{B} \frac{\partial C}{\partial y}=h_{2}\left(C_{f}-C\right) a t y=0 \\
u \rightarrow 0, T \rightarrow T_{\infty}, C \rightarrow C_{\infty}, \text { as } y \rightarrow \infty
\end{gathered}
$$

The convective surface concentration and temperature are represented by $C_{f}$ and $T_{f}$ respectively, $h_{1}=a x^{(n-1) / 2}, h_{2}=c x^{(n-1) / 2}$ depicts the coefficient of heat and mass transfer respectively, the term $V_{w}=V_{0} x^{(n-1) / 2}$ describes the suction/injection, where $a, c$ and $V_{0}$ are constant. Various symbols used in this research are given in Table.1.

The dimensionless variables mentioned below are inserted into the main equations.

$$
\eta=\sqrt{\frac{b(n+1) x^{n-1}}{2 v}} y, \psi=\sqrt{\frac{2 v b x^{n+1}}{n+1}} f(\eta), \theta(\eta)=\frac{T-T_{\infty}}{T_{f}-T_{\infty}}, h(\eta)=\frac{C-C_{\infty}}{C_{f}-C_{\infty}}, u=\frac{\partial \psi}{\partial y}, v=-\frac{\partial \psi}{\partial x}
$$

The continuity Eq. (6) becomes true when the quantities in Eq. (11) are substituted into the governing Eqs. (6-9), while Eqs. (7-9) yield the under listed:

$$
\begin{gathered}
f^{\prime \prime \prime}+W e f^{\prime \prime} f^{\prime \prime \prime}-\frac{2 n}{n+1} f^{\prime 2}+f^{\prime \prime}-\frac{2}{n+1} M_{0} f^{\prime}+\frac{2}{n+1} H e^{(-B \eta)}+\frac{2}{n+1} \lambda_{1}(\theta-R h)=0 \\
\frac{1}{P_{r}}\left[1+\delta \theta+N_{r}\left(1+\left(\theta_{b}-1\right) \theta\right)^{3}\right] \theta^{\prime \prime}+\frac{3}{P_{r}}\left[N_{r}\left(\theta_{b}-1\right)\left(1+\left(\theta_{b}-1\right) \theta\right)^{2}\right] \theta^{\prime 2}+\delta \theta^{\prime 2}+f \theta^{\prime}+N_{t} \theta^{\prime 2}+N_{b} \theta^{\prime} h^{\prime}=0 \\
h^{\prime \prime}+S c\left(f h^{\prime}-\frac{2}{n+1} \gamma_{1} h\right)+\frac{N_{t}}{N_{b}} \theta^{\prime \prime}=0
\end{gathered}
$$

The dimensionless boundary conditions are

$$
f^{\prime}(0)=1, f(0)=F_{w}, \theta^{\prime}(0)=-B_{1}(1-\theta(0)), h^{\prime}(0)=-B_{2}(1-h(0)), f^{\prime}(\infty)=0, \theta(\infty)=0, h(\infty)=0
$$

The entropy generation equation. The volumetric rate of entropy generation for the Williamson fluid influenced by nonlinear thermal radiation and diffusion effect is modeled using thermodynamics second law

$$
S_{G n}=S_{h t}+S_{d}
$$




\begin{tabular}{|l|l|l|l|}
\hline Parameter & Description & Parameter & Description \\
\hline$W e$ & Material parameter & $\lambda_{1}$ & Mixed convection term \\
\hline$\theta_{b}$ & Temperature ratio term & $R$ & Buoyancy ratio term \\
\hline$N_{r}$ & Radiation term & $\theta_{r}$ & Wall temperature excess ratio \\
\hline$\gamma_{1}$ & Chemical reaction & $P_{r}$ & Prandtl number \\
\hline$\gamma_{2}$ & Diffusion Constant & $H$ & Modified Hartmann number \\
\hline$N_{t}$ & Thermophoresis parameter & $\delta$ & Thermal conductivity term \\
\hline$B_{1}$ & Thermal Biot number & $D a$ & Darcy number \\
\hline$B_{2}$ & Mass Biot number & $S c$ & Schmidt number \\
\hline$B_{r}$ & Brikman number & $N_{b}$ & Brownian motion parameter \\
\hline
\end{tabular}

Table 2. lists of the evolving physical quantities that join the dimensionless governing equations.

where $S_{G n}$ denotes volumetric entropy generation, $S_{h t}$ denotes entropy generation of heat transfer or conduction and $S_{d}$ denotes entropy generation of mass transfer effects over a finite temperature and concentration difference. As a result, $S_{G n}$ can be written as:

$$
S_{G n}=\frac{1}{T^{2}}\left(k+\frac{16 a^{*} T^{3}}{3 b^{*} k}\right)\left(\frac{\partial T}{\partial y}\right)^{2}+\left[\frac{R D_{B}}{C}\left(\frac{\partial C}{\partial y}\right)^{2}+\frac{R D_{B}}{T}\left(\frac{\partial C}{\partial y} \frac{\partial T}{\partial y}\right)\right]
$$

For the applied boundary conditions (10), the characteristic entropy generation $S_{G}^{\prime \prime \prime}$ is given as

$$
S_{G}^{\prime \prime \prime}=k_{\infty} \frac{\left(T_{w}-T_{\infty}\right)^{2}(n+1)}{2 T_{\infty}^{2} x^{2}}
$$

The generation of dimensionless entropy can be written as

$$
N_{G s}=\frac{\operatorname{Re}\left[1+\delta \theta+N_{r}\left(1+\left(\theta_{b}-1\right) \theta\right)^{3}\right]}{\left(1+\left(\theta_{b}-1\right) \theta\right)^{2}} \theta^{\prime 2}+\frac{\operatorname{Re} \gamma_{2}\left(C_{b}-1\right)}{\left(\theta_{b}-1\right)}\left[\frac{\left(C_{b}-1\right) C^{\prime 2}}{\left(1+\left(C_{b}-1\right) C\right)\left(\theta_{b}-1\right)}+\frac{\theta^{\prime} C^{\prime}}{1+\left(\theta_{b}-1\right) \theta}\right]=0
$$

The entropy output number is represented by the word $N_{G s}=\frac{S_{G n}}{S_{G}^{\prime \prime}}=N_{h}+N_{m} . N_{h}$ is the first term in the RHS of Eq. (19), which denotes entropy generation caused by heat transfer, while $N_{m}$ denotes entropy generation caused by mass transfer.

Bejan number. The entropy generation number $N_{G s}$ determines the distribution of entropy generation in the flow area, but in an energy optimization problem, the contribution of thermal conductivity to total entropy output is needed. The Bejan number (Be) expresses the value of thermal irreversibility in comparison to absolute irreversibility and is used to describe a number.

$$
B e=\frac{N_{h}}{N_{G s}}
$$

The emerging parameters from the governing equations are described as:

$$
\begin{aligned}
\theta_{b} & =\frac{T_{f}}{T_{\infty}}, W e=\frac{\Gamma}{2} \sqrt{\frac{b^{3}(n+1) x^{3 n-1}}{v}}, M_{0}=\frac{\sigma B_{0}^{2} x}{b \rho}, H=\frac{\pi j_{0} M^{*}}{8 \rho_{f} b^{2} x^{2 n-1}}, \\
B & =\frac{\pi}{s} \sqrt{\frac{2 v}{b(n+1) x^{n+1}}}, \lambda_{1}=\frac{g \beta_{T}\left(1-C_{\infty}\right) \rho_{f}\left(T_{f}-T_{\infty}\right)}{b^{2} \rho_{f} x^{2 n-1}}, R=\frac{\left(\rho_{p}-\rho_{\infty}\right)\left(C_{f}-C_{\infty}\right)}{\beta_{T}\left(1-C_{\infty}\right)\left(T_{f}-T_{\infty}\right) \rho_{f}}, \\
P_{r} & =\frac{\mu_{f} c_{p}}{k_{\infty}}, N_{r}=\frac{16 a^{*} T_{\infty}^{3}}{3 b^{*} k_{\infty}} N_{b}=\frac{(\rho c p)_{p} D_{B}\left(C_{\omega}-C_{\infty}\right)}{v_{f}(\rho c p)_{f}}, \\
R e & =\frac{b x^{n-1}}{v_{f}}, B_{1}=\frac{a}{k_{\infty}} \sqrt{\frac{2 v}{b(n+1)}}, B_{2}=\frac{c}{D_{B}} \sqrt{\frac{2 v}{b(n+1)}}, N_{t}=\frac{(\rho c p)_{p} D_{T}\left(T_{\omega}-T_{\infty}\right)}{v_{f}(\rho c p)_{f}}
\end{aligned}
$$

The modeled parameter after simplification are given in Table 2.

Engineers are particularly interested in the skin friction coefficient $c_{f}$, the local Nusselt number $N_{u}$ and the local Sherwood number $S_{h}$, which are all ordered as

$$
c_{f}=\frac{\tau_{\omega}}{\rho U_{\omega}^{2}}, N_{u}=\frac{x q_{\omega}}{k\left(T_{f}-T_{\infty}\right)}, S_{h}=\frac{x q_{m}}{D_{B}\left(C_{f}-C_{\infty}\right)}
$$




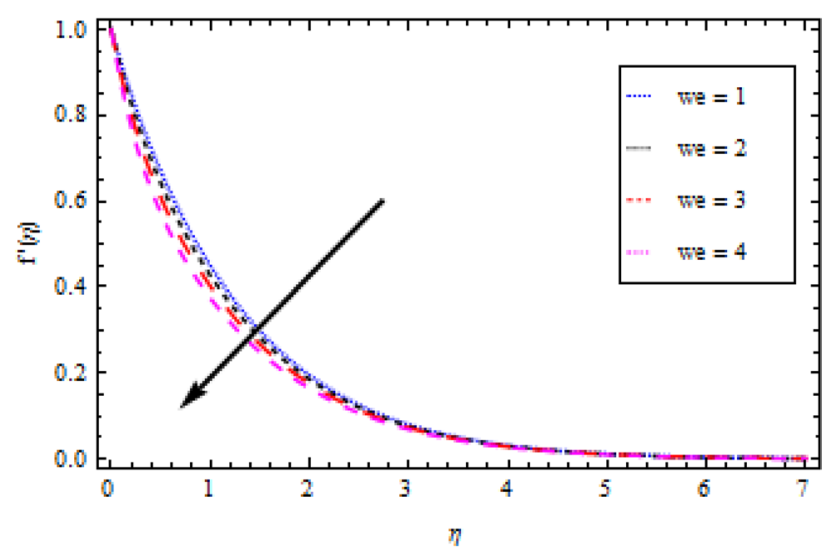

Figure 2. Velocity profile variation with respect to We.

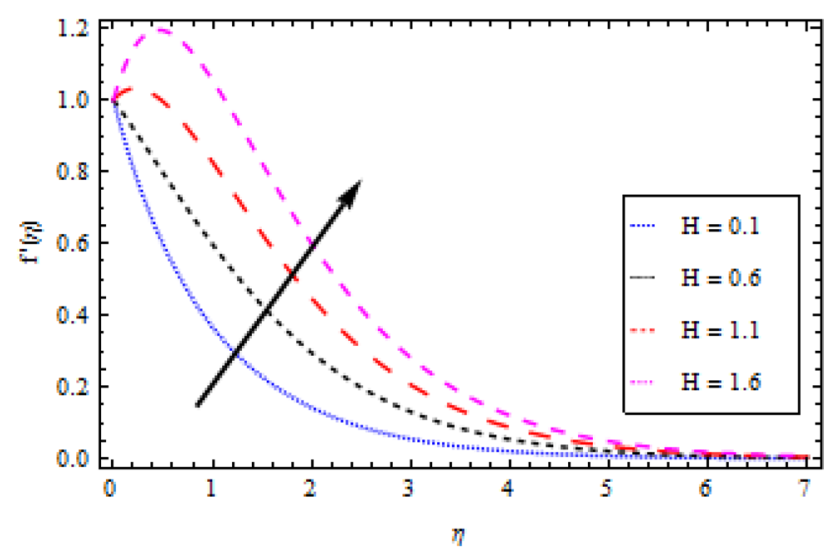

Figure 3. Velocity profile variation with respect to $H$.

where $\tau_{\omega}, q_{\omega}$ and $q_{m}$ are defines the shear stress, heat flux and mass flux at the surface respectively. These are expressed as

$$
\tau_{\omega}=\left.v\left(\frac{\partial u}{\partial y}+\frac{1}{2} \nu \Gamma\left(\frac{\partial u}{\partial y}\right)^{2}\right)\right|_{y=0}, q_{m}-\left.\left(k+\frac{16 T^{3} a^{*}}{3 k b^{*}}\right) \frac{\partial T}{\partial y}\right|_{y=0}, q_{m}=-\left.D_{B}\left(\frac{\partial C}{\partial y}\right)\right|_{y=0}
$$

In view of Eqs. (11) and (23), the quantities in (22) respectively yields (24-26)

$$
\begin{gathered}
\tau_{\omega}=\left(\frac{n+1}{2}\right)^{1 / 2}\left(f^{\prime \prime}(0)+\frac{W e}{2}\left(f^{\prime \prime}(0)\right)^{2}\right) R e^{-1 / 2} \\
N_{u}=-\left(\frac{n+1}{2}\right)^{1 / 2}\left(1+N_{r}\left(1+\left(\theta_{r}-1\right) \theta(0)\right)^{3}\right) R e^{1 / 2} \theta^{\prime}(0) \\
S_{h}=-\left(\frac{n+1}{2}\right)^{1 / 2} R e^{1 / 2} h^{\prime}(0)
\end{gathered}
$$

\section{Result and discussion}

Velocity profile. In this part, we'll look at how fluid parameters affect the flow profile. Significant importance is given to fluid parameters such as modified Hartman factor $H$, dimensionaless parameter $B$, fluid parameter $W e$, magnetic interaction parameter $M_{0}$, heat and mass Biot numbers $B_{1}, B_{2}$, Brownian motion factor $N_{b}$, thermophoretic parameter $N_{t}$, Brikman number $B_{r}$ and Buoyancy ratio $R$. Figures 2, 3, 4, and 5 show how $W e, H, M_{0}$ and $B$ affect the velocity profile. The flow profile is greatly fall down when the Williamson parameter $W e$ is used. We values that are higher continue to improve the velocity profile. In the presence of multiple dimensionless parameters, the plot of describing the velocity field against $\eta$ for various values of $H$ indicates that the 


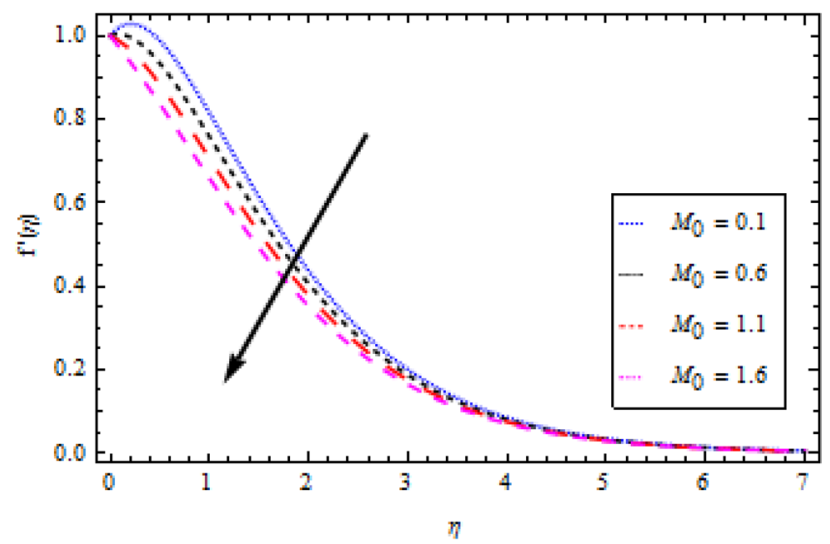

Figure 4. Velocity profile variation with respect to $M_{0}$.

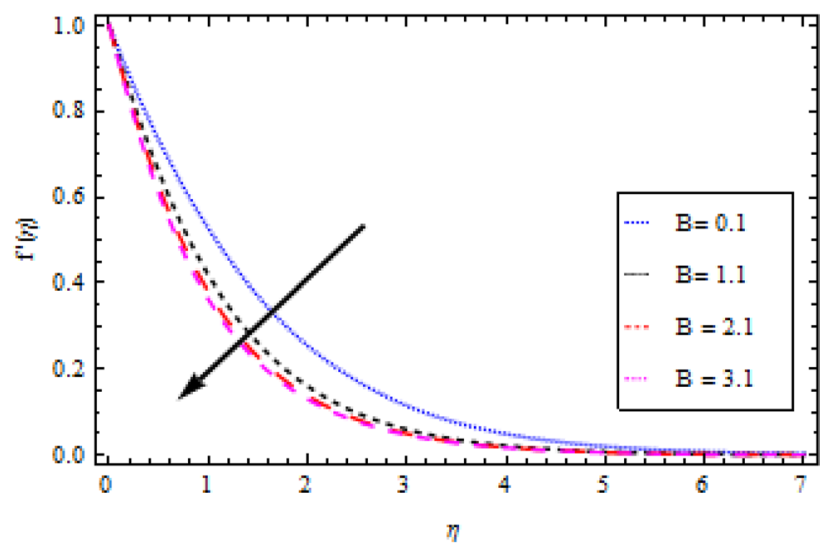

Figure 5. Velocity profile variation with respect to $B$.

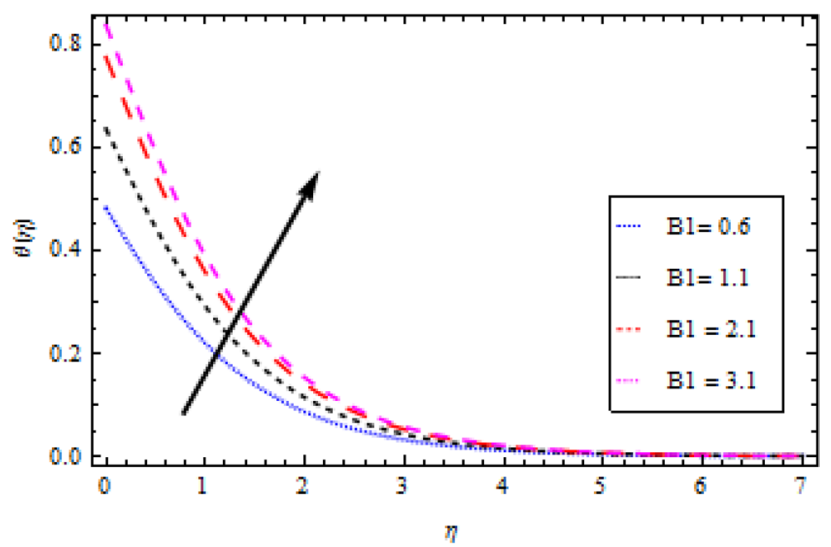

Figure 6. Temperature profile variation with respect to $B_{1}$.

velocity field accelerates with an enlargement in $H$, as shown in Fig. 3. Actually, this the pattern corresponds to the problem's physical principles in the sense that $H>0$ denotes an assisting flow mechanism on the velocity region. Figure 4 demonstrates the influence of magnetic parameter $M_{0}$ over velocity profile. It is noticed that the velocity reduces by increasing $M_{0}$. As shown in Fig. 5, as the dimensionless parameter $B$ is increased, the fluid motion decreases.

Temperature profile. The Plots of different fluid factors on the thermal profile are shown in Figs. 6, 7, 8, 9 and 10. Figure 6 depicts the reaction of the surface convection term Biot number $B_{1}$ on the thermal sector. As a 


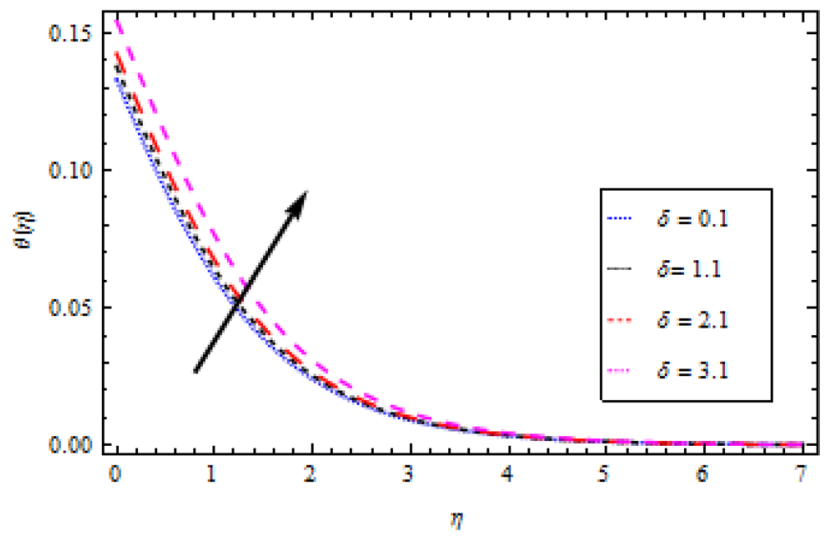

Figure 7. Temperature profile variation with respect to $\delta$.

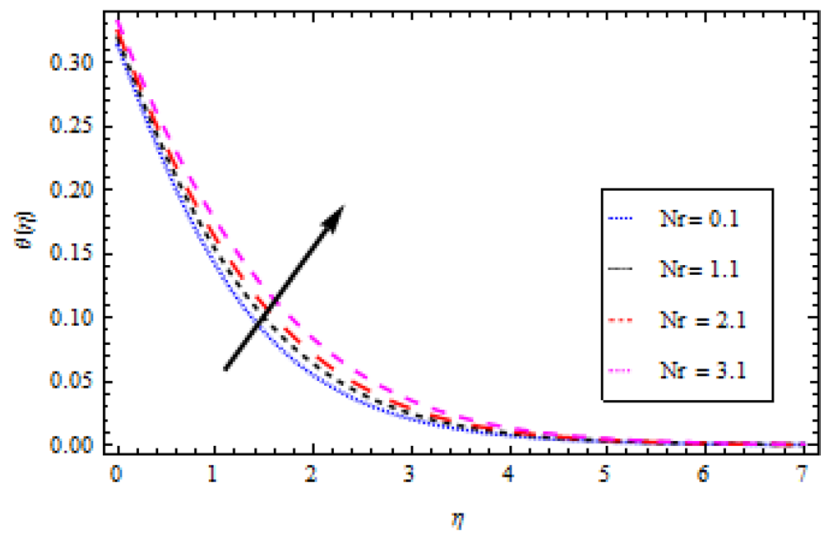

Figure 8. Temperature profile variation with respect to $N_{r}$.

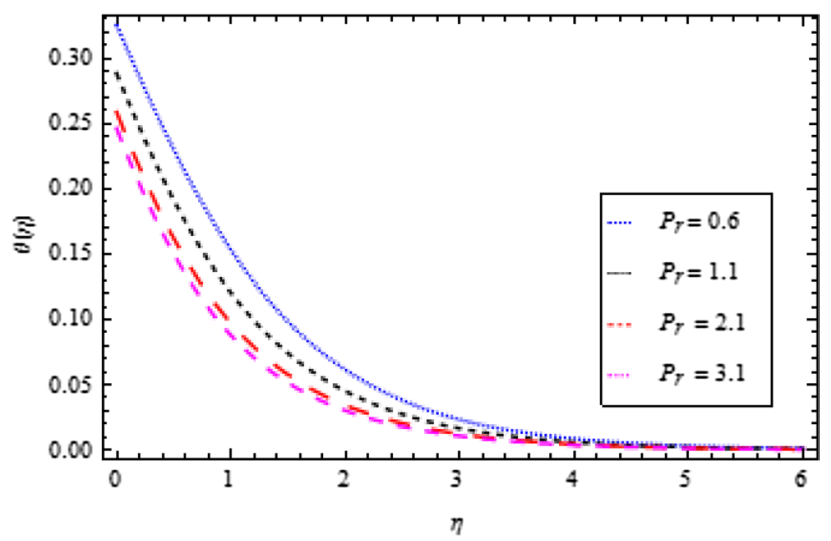

Figure 9. Temperature profile variation with respect to $P_{r}$.

result of the escalation in $B_{1}$, the size of the thermal boundary layer expands and the temperature distribution increases, as seen in the figure. A rise in $B_{1}$ strengthens the heat coefficient transport, causing the temperature field to rise. Similarly, with an increase in the magnitude of thermal conductivity term $\delta$, the temperature field improves as demonstrated in Fig. 7. The outcome of the radiation parameter $N_{r}$ on the thermal profile is shown in Fig. 8. Due to a durable heat source, increased values of the radiation parameter result in an escalation in temperature. The temperature profile is depicted in Fig. 9 directly influenced by the momentum to mass diffusivity ratio. When the involved parameter $P_{r}$ is augmented, a decreasing behavior is observed. A growth in temperature ratio $\theta_{b}$ improves the surface temperature as illustrated in Fig. 10. 


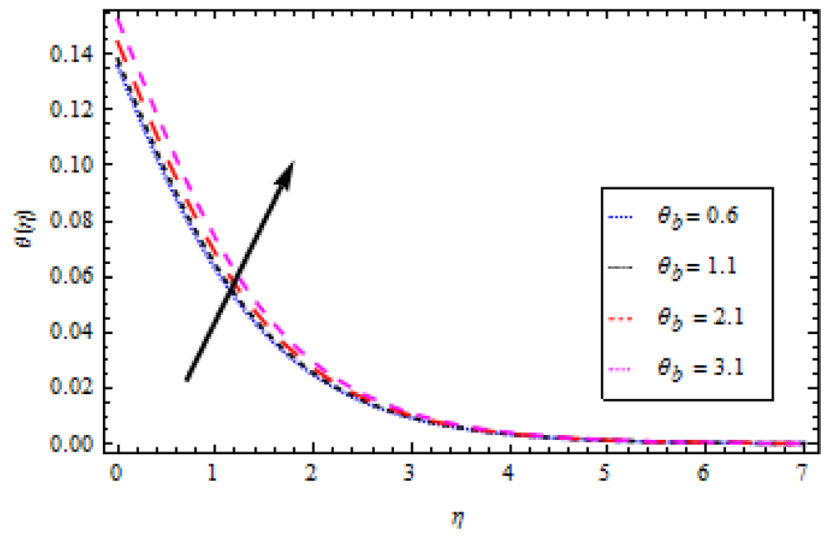

Figure 10. Temperature profile variation with respect to $\theta_{b}$.

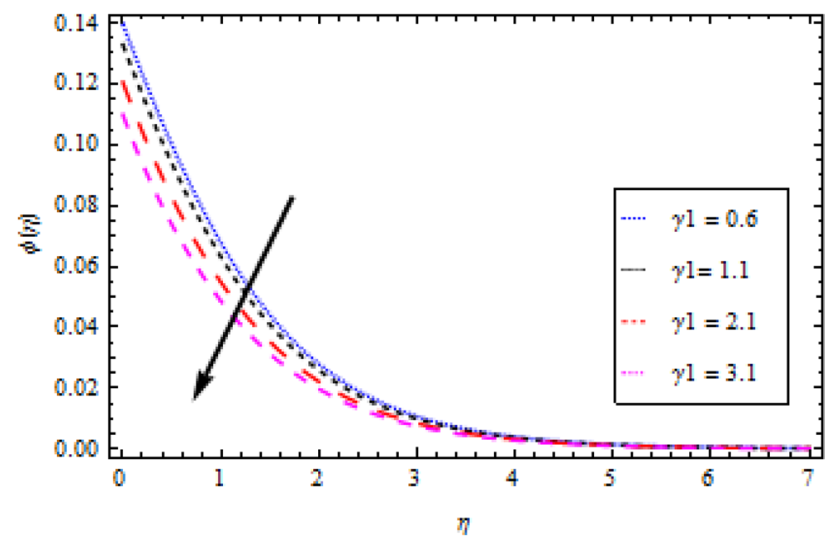

Figure 11. Concentration profile variation with to $\gamma_{1}$.

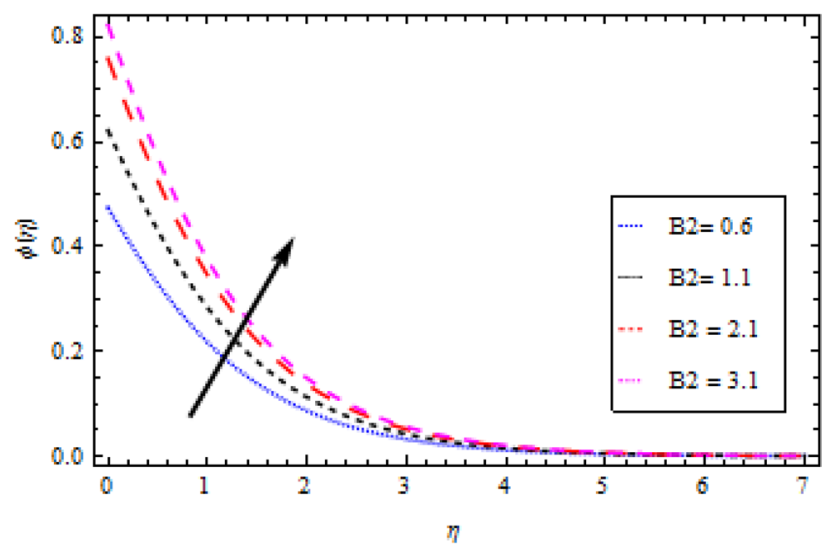

Figure 12. Concentration profile variation with respect to $B_{2}$.

Concentration profile. The graphs in Figs. 11, 12, 13, 14 and 15 show different factors alongside the concentration profile. In Fig. 11 the impression of the chemical reaction factor $\gamma_{1}$ on the concentration field is described. It has been discovered that increasing $\gamma_{1}$ causes the concentration boundary layer to shrink, lowering the concentration profile. As shown in Fig. 12, the concentration boundary structure is associated with rising values of the mass Biot number $B_{2}$. Figure 13 shows a plot of the concentration profile versus $\eta$ for Schmidt number $S c$ variation. With an upturn in $S c$, the concentration area alongside the solutes boundary layer thins out. Similarly, Fig. 14 shows a plot of the concentration profile for thermoporesis parameter $N_{t}$ variation. The concentration profile decreases by increasing $N_{t}$. The outcome of the Brownian motion $N_{b}$ on the concentration 


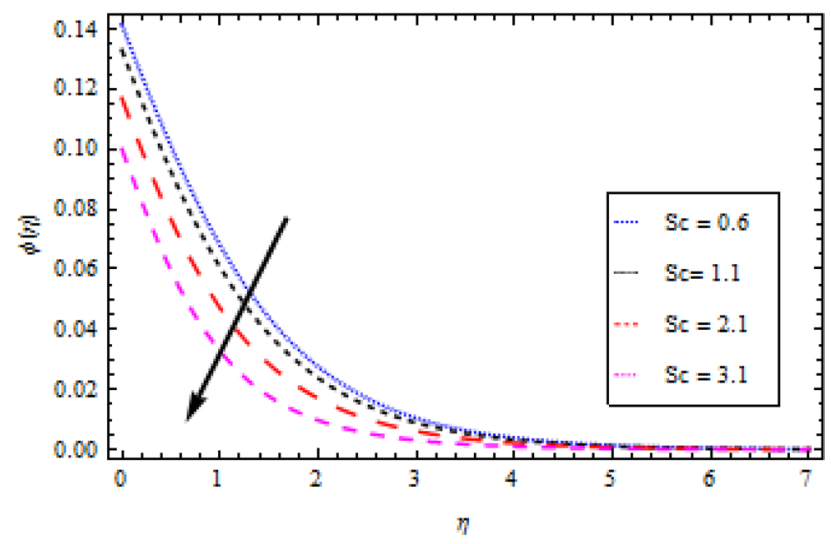

Figure 13. Concentration profile variation with respect to $S c$.

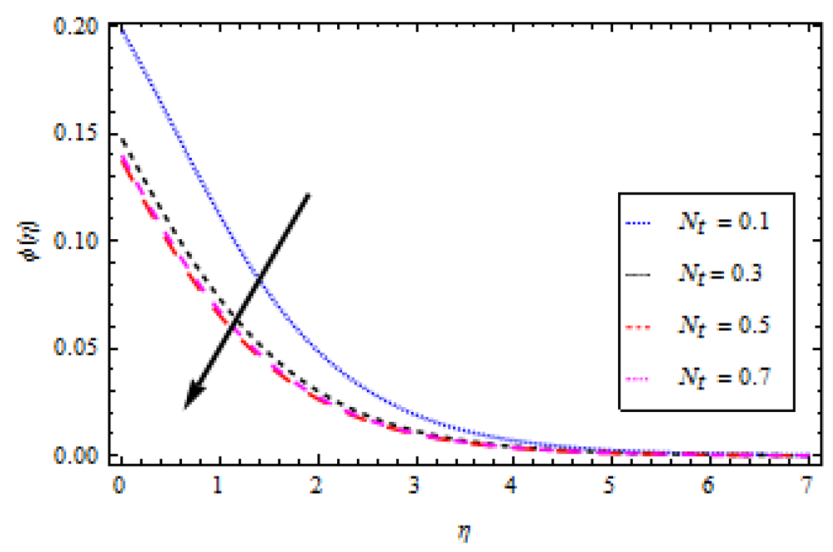

Figure 14. Concentration profile variation with respect to $N_{t}$.

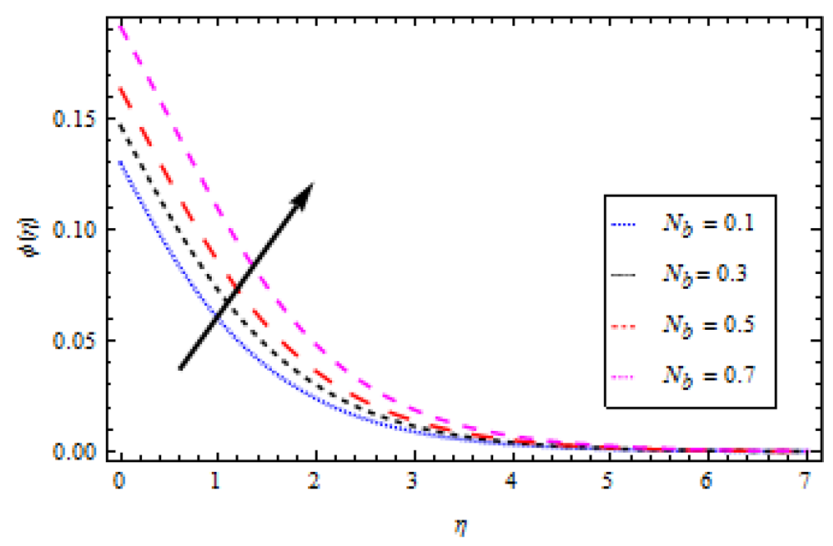

Figure 15. Concentration profile variation with respect to $N_{b}$.

profile is plotted in Fig. 15. The figure shows that the concentration boundary layer is associated with increasing values of $N_{b}$.

Entropy generation. Figures 16, 17 and 18 are show graphs of different parameters versus entropy generation. The reaction of entropy generation number $N_{G s}$ to changes in temperature ratio $\theta_{b}$ values are depicted in Fig. 16. An uplift in $\theta_{b}$ decreases $N_{G s}$. With an increase in $N_{r}$, entropy generation $N_{G s}$ also increases, as shown in Fig. 17, which specifies that heat transmission irreversibility dictates entropy generation. It is noticed that 


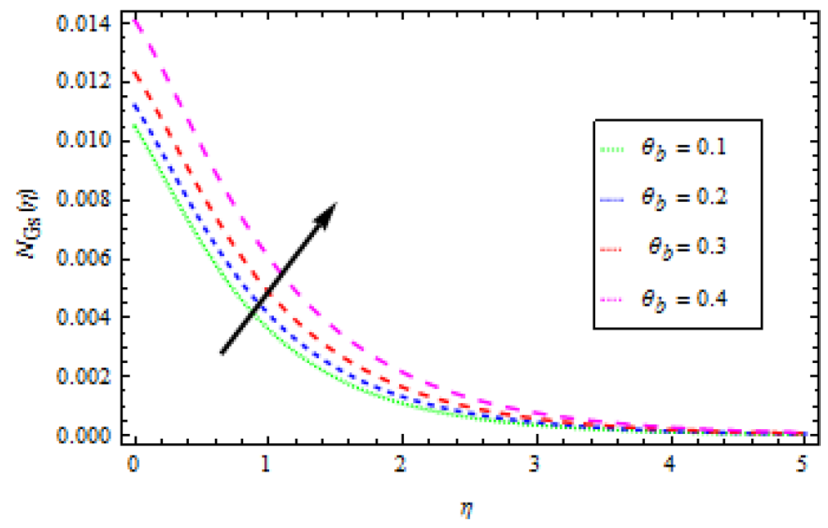

Figure 16. Effects of $\theta_{b}$ on entropy generation.

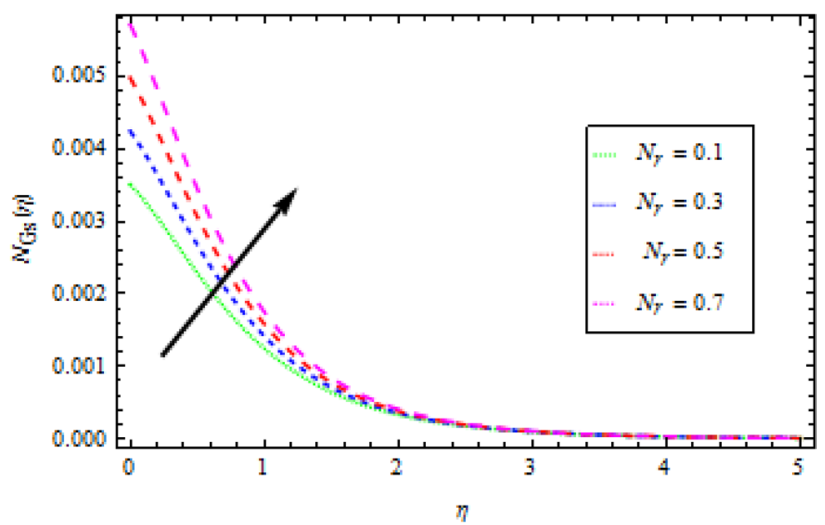

Figure 17. Effects of $N_{r}$ on entropy generation.

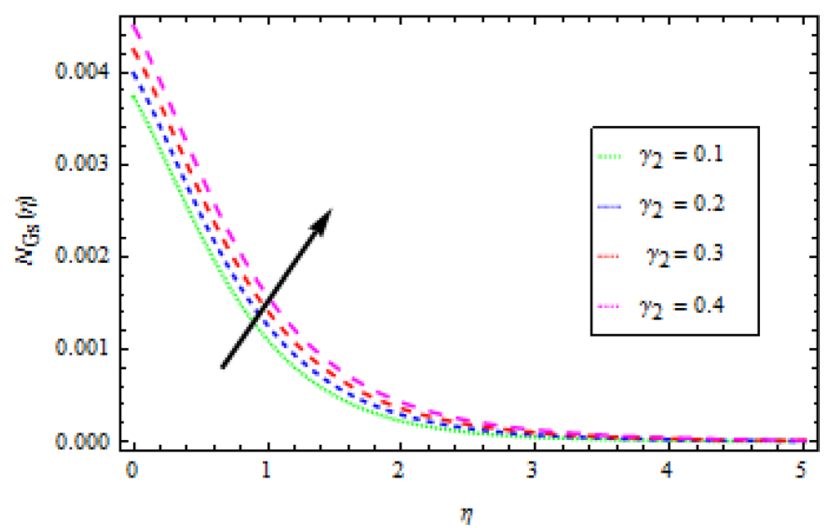

Figure 18. Effects of $\gamma_{2}$ on entropy generation.

increase in radiative parameter increases Bejan number. In Fig. 18, the effect of the diffusion constant $\gamma_{2}$ on entropy generation $N_{G s}$ is highlighted. It has been observed that as $\gamma_{2}$ increases, $N_{G s}$ increases.

Bejan number. Figures 19 and 20 show the effects of $N_{r}$ and $\theta_{b}$ on the Bejan number $B e$, respectively. Be improves with an increase in both $N_{r}$ and $\theta_{b}$, as seen in these graphs. The assumption is that as the values of $N_{r}$ and $\theta_{b}$ rise, the entropy generated by heat and mass transfer outnumbers that are produced by frictional heating. 


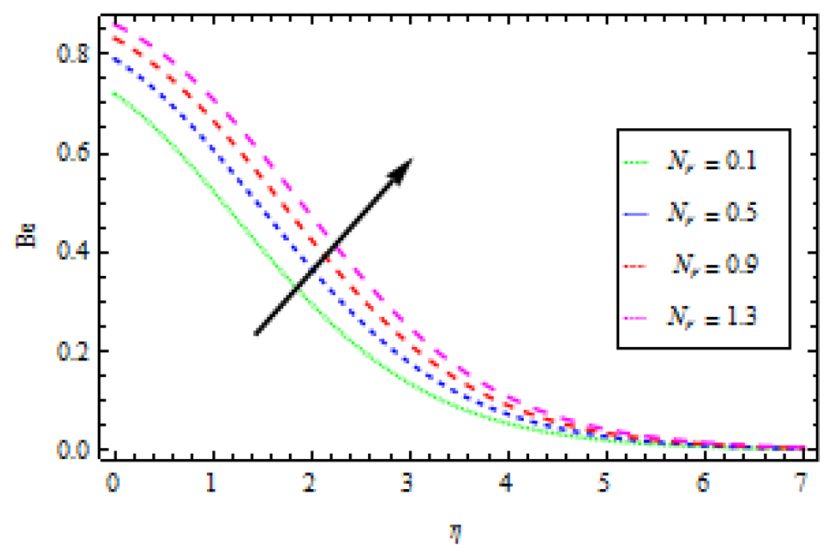

Figure 19. Effects of $N_{r}$ on Bejan number.

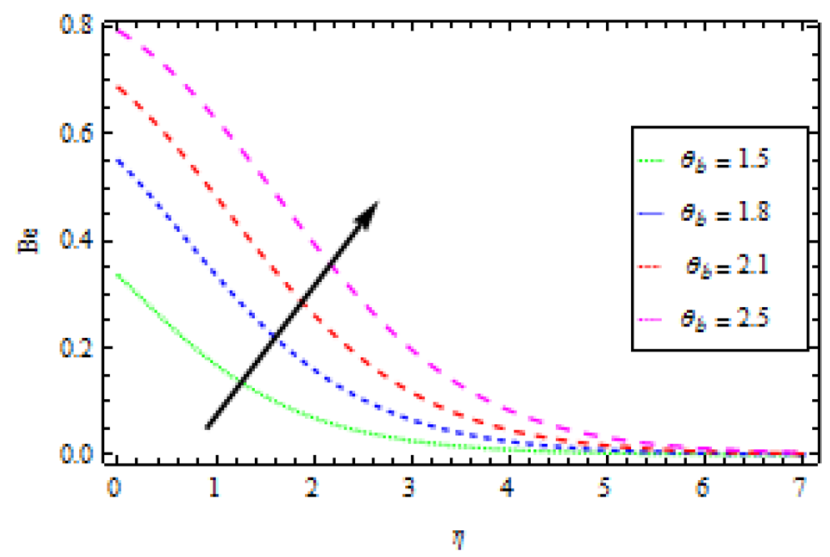

Figure 20. Effects of $\theta_{b}$ on Bejan number.

\section{Conclusion}

Entropy production in a reactive Williamson nanofluid MHD movement over a vertical Riga plate has been studied using an analytical solution. The effects of nonlinear thermal radiation, thermophoresis, Brownian motion and varying thermal conductivity are all included in the model. The parametric effects of emerging terms on non-dimensional quantities are graphically represented and discussed as a result. According to the report:

- The velocity profile rises as the fluid parameter $W e$, magnetic parameter $M_{0}$ and dimensionless number $B$ are increased. As the velocity profile decreases, the modified Hartman number $H$ rises.

- With the increase in surface convection term (Biot number) $B_{1}$, thermal conductivity $\delta$, radiation term $N_{r}$ and temperature ratio $\theta_{b}$, the thermal field expands. While the thermal field shrink with incremental values of Prandtl number.

- By rising the values of mass Biot number $B_{2}$ and Brownian motion factor $N_{b}$, the concentration profile rises. However, for raising the values of chemical reaction $\gamma_{1}$, Schmidt number $S c$ and thermoporesis parameter $N_{t}$, the opposite behaviour was observed.

- With the increase in radiation term $N_{r}$ and temperature ratio parameter $\theta_{b}$, the entropy generation is high, while opposite behaviour was noticed when diffusion constant term $\gamma_{2}$ increased.

- Bejan number rises as the radiation term $N_{r}$ and temperature ratio $\theta_{b}$ rise.

Received: 15 June 2021; Accepted: 30 August 2021

Published online: 15 September 2021

\section{References}

1. Choi, S. U. S. \& Eastman, J. A. Enhancing thermal conductivity of fluids with nanoparticles. ASME Int. Mech. Eng. Congr. Expos. 231, 12-17 (1995)

2. Buongiorno, J. Convective transport in nanofluid. J. Heat Transf. 128, 240-250 (2006).

3. Mustafa, M., Hayat, T., Pop, I., Asghar, S. \& Obaidat, S. Stagnation-point flow of a nanofluid towards a stretching sheet. Int. J. Heat Mass Transf. 54, 5588-5594 (2011). 
4. Turkyilmazoglu, M. Exact analytic solutions for heat and mass transfer of MHD slip flow in nanofluids. Chem. Eng. Sci. 84, 182-187 (2012).

5. Kuznetsov, A. V. \& Nield, D. A. The Cheng-Minkowycz problem for natural convective boundary layer flow in a porous medium saturated by a nanofluid: a revised model. Int. J. Heat Mass Transf. 65, 682-685 (2013).

6. Hsiao, K. L. Nanofluid flow with mutimedia physical features for conjugate mixed convection and radiation. Comput. Fluids 104, $1-8(2013)$.

7. Malvandi, A. \& Ganji, D. D. Brownian motion and thermophoresis effects on slip flow of alumina/water nanofluid inside a circular microchannel in the pressure of a magnetic field. Int. J. Therm. Sci. 84, 196-206 (2014).

8. Gailitis, A. \& Lielausis, O. On a possibility to reduce the hydrodynamic resistance of a plate in an electrolyte. App. Magn. Rep. Phys. Inst. Riga 12, 143-146 (1961).

9. Ahmad, R., Mustafa, M. \& Turkyilmazoglu, M. Buoyancy effects on nanofluid flow past a convectively heated vertical Riga-plate: A numerical study. Int. J. Heat and Mass Transf. 111, 827-835 (2017).

10. Sheikholeslami, M. \& Chamkha, A. J. Influence of Lorentz forces on nanofluid forced convection considering Marangonai convection. J. Mol. Liq. 225, 750-757 (2017).

11. Shafiq, A., Hammouch, Z. \& Turab, A. Impact of radiation in a stagnation point flow of Walters' B fluid towards a Riga plate. Therm. Sci. Eng. Progr. 6, 27-33 (2018).

12. Adeel, A., Saleem, A. \& Sumaira, A. Flow of nanofluid past a Riga plate. J. Magn. Magn. Mater. 402, 44-48 (2016).

13. Rasool, G., Zhang, T., Shafiq, A. \& Durur, H. Influence of chemical reaction on Marangoni convective flow of nanofluid in the presence of Lorentz forces and thermal radiation: A numerical investigation. J. Adv. Nanotechnol. 1(1), 32-49 (2019).

14. Williamson, R. V. The flow of pseudoplastic materials. Ind. Eng. Chem. Res. 21(11), 1108-1111 (1929).

15. Lyubimov, D. V. \& Perminov, A. V. Motion of a thin oblique layer of a pseudoplastic fluid. J. Eng. Phys. Thermophys. 75(4), 920-924 (2002).

16. Dapra, I. \& Scarpi, G. Perturbation solution for pulsatile flow of a non-Newtonian Williamson fluid in a rock fracture. Int. J. Rock Mech. Mining Sci. 44, 271-278 (2007).

17. Nadeem, S. \& Akram, S. Peristaltic flow of a Williamson fluid in an asymmetric channel. Commun. Nonlinear Sci. Numer. Simul. 15, 1705-1716 (2010).

18. Vasudev, C., Rao, U. R., Reddy, M. V. S. \& Rao, G. P. Peristaltic pumping of Williamson fluid through a porous medium in horizontal channel with heat transfer. Am. J. Sci. Ind. Res. 1(3), 656-666 (2010).

19. Nadeem, S., Hussain, S. T. \& Lee, C. Flow of a Williamson fluid over a stretching sheet. Braz. J. Chem. Eng. 30(03), 619-625 (2013).

20. Cramer, S. D. \& Marchello, J. M. Numerical evaluation of models describing non-Newtonian behavior. Am. Inst. Chem. Eng. J. 14, 980-983 (1968).

21. Khan, J. A., Mustafa, M., Hayat, T. \& Turkyilmazoglu, M. Numerical study of nanofluid flow and heat transfer over a rotating disk using Buongiorno's model. Int. J. Numer. Methods Heat Fluid Flow 27(1), 221-234 (2007).

22. Turkyilmazoglu, M. Magnetic field and slip effects on the flow and heat transfer of stagnation point Jeffrey fluid over deformable surfaces. Zeit. Fur Naurf. A 71(6), 549-556 (2016).

23. Abo-Alkhair, R. E., Bhatti, M. M. \& Mekheimer, K. S. Magnetic force effects on peristaltic transport of hybrid bionanofluid ( $\mathrm{Au}-\mathrm{Cu}$ nanoparticles) with moderate Reynolds number: An expanding horizon. Int. Commun. Heat Mass Trans. 123, 105228 (2021).

24. Bhatti, M. M., Al-Khaled, K., Khan, S. U., Chammam, W. \& Awais, M. Darcy-Forchheimer higher order slip flow of Eyring-Powell nanofluid with nonlinear thermal radiation and biconvection phenomenon. J. Disp. Sci. Technol. https://doi.org/10.1080/01932 691.2021.1942035 (2021).

25. Turkyilmazoglu, M. On the transparent effects of Buongirono nanofluid model on heat and mass transfer. Eur. Phys. J. Plus 136, $376(2021)$

26. Turkyilmazoglu, M. Multiple analytical solution of heat and mass transfer of magnetohydrodynamic slip flow for two types of viscoelastic fluids over a stretching surface. J. Heat Transf. 134(7), 071701 (2012).

27. Turkyilmazoglu, M. Nanoliquid film flow due to a moving substrate and heat transfer. Eur. Phys. J. Plus 135, 781 (2020).

28. Turkyilmazoglu, M. Latitudinally deforming rotating sphere. App. Math. Model. 71, 1-11 (2019).

29. Zhang, L. et al. Nonlinear nanofluid flow under the consequences of Lorentz forces and Arrhenius kinetics through a permeable surface: A robust spectral approach. J. Taiwan Inst. Chem. Eng. 124, 98-105 (2021).

30. Zhang, L., Nazar, T., Bhatti, M. M. \& Michaelides, E. E. Stability analysis on the kerosene nanofluid flow with hybrid zinc/ aluminum-oxide $\left(\mathrm{ZnO}-\mathrm{Al}_{2} \mathrm{O}_{3}\right)$ nanoparticles under Lorentz force. Int. J. Numer. Methods Heat Fluid Flow https://doi.org/10.1108/ HFF-02-2021-0103 (2021).

31. Bhatti, M. M., Zeeshan, A., Asif, M. A., Ellahi, R. \& Sadiq, M. S. Non-uniform pumping flow model for the couple stress particlefluid under magnetic effects. Chem. Eng. Commun. https://doi.org/10.1080/00986445.2021.1940156 (2021).

\section{Acknowledgements}

"The authors acknowledge the financial support provided by the Center of Excellence in Theoretical and Computational Science (TaCS-CoE), KMUTT". Moreover, this research project is supported by Thailand Science Research and Innovation (TSRI) Basic Research Fund: Fiscal year 2021 under project number 64A306000005.

\section{Author contributions}

M.R. and Z.S. modeled and solved the problem. M.A.J. and M.R. wrote the manuscript. P.K., and A.S. contributed in the numerical computations and plotting the graphical results. All authors finalized the manuscript after its internal evaluation.

\section{Competing interests}

The authors declare no competing interests.

\section{Additional information}

Correspondence and requests for materials should be addressed to Z.S. or P.K.

Reprints and permissions information is available at www.nature.com/reprints.

Publisher's note Springer Nature remains neutral with regard to jurisdictional claims in published maps and institutional affiliations. 
(c) (i) Open Access This article is licensed under a Creative Commons Attribution 4.0 International cc) License, which permits use, sharing, adaptation, distribution and reproduction in any medium or format, as long as you give appropriate credit to the original author(s) and the source, provide a link to the Creative Commons licence, and indicate if changes were made. The images or other third party material in this article are included in the article's Creative Commons licence, unless indicated otherwise in a credit line to the material. If material is not included in the article's Creative Commons licence and your intended use is not permitted by statutory regulation or exceeds the permitted use, you will need to obtain permission directly from the copyright holder. To view a copy of this licence, visit http://creativecommons.org/licenses/by/4.0/.

(C) The Author(s) 2021 